\title{
Versatility of Adipofascial Anterolateral Thigh Flap for Reconstruction of Maxillary Defects with Infratemporal Fossa Extension
}

\author{
Vikram D. Kekatpure, MS, MCh ${ }^{1}$ Naveen Hedne, MS, MCh ${ }^{1}$ Sachin Chavre, DNB (Plastic Surgery) ${ }^{1}$ \\ Vijay Pillai, MDS, MBBS ${ }^{1}$ Nirav Trivedi, MS, MCh ${ }^{1}$ Moni Abraham Kuriakose, MD, FRCS ${ }^{1}$ \\ ${ }^{1}$ Department of Head and Neck Oncology, Mazumdar Shaw Cancer \\ Center, Narayana Hrudayalaya, Bangalore, Karnataka, India \\ Craniomaxillofac Trauma Reconstruction 2014;7:213-217

\begin{abstract}
Address for correspondence Vikram D. Kekatpure, MS, MCh, Department of Head and Neck Oncology, Mazumdar Shaw Cancer Center, Narayana Hrudayalaya, 256/A, Bommasandra Industrial Area, Bangalore
\end{abstract} \\ 560099, Karnataka, India (e-mail: kvikram2000@gmail.com).
}

\begin{abstract}
Keywords

- adipofascial flap

- infratemporal fossa

- maxillectomy defects

- anterolateral thigh flap

Tumors arising from the posterior hard palate or posterolateral maxilla may extend to involve the infratemporal fossa (ITF). Resection of these tumors results in infrastructural maxillectomy with ITF defects. In this study, we describe the use of an adipofascial anterolateral thigh flap (ALT) specifically for such defects. This case series includes four patients who underwent an infrastructure maxillectomy with ITF clearance and the resultant defects were reconstructed using adipofascial anterolateral thigh flaps. The complications as well as the functional outcomes were assessed. This study included patients with lesions involving the hard palate, posterolateral part of maxilla with extension into the ITF. The mean flap dimension was $150 \mathrm{~cm}^{2}$ (range, $120-180 \mathrm{~cm}^{2}$ ). All flaps were harvested based on a single perforator. The flap was used to obliterate the ITF defect and also to achieve oroantral separation. All flaps mucosalized well within 6 weeks. All patients were on oral diet and had adequate mouth opening. There were no donor-site complications. Adipofascial ALT is an excellent choice for infrastructural maxillectomy defects with ITF extension. The intraoral part got mucosalized well and provided a smooth and taut surface. A large adipofascial tissue flap helps obliterate the ITF, thus minimizing complications.
\end{abstract}

The postablative defects of the midface have significant functional and cosmetic consequences. To achieve an optimal reconstructive algorithm, multiple defect-based classifications have been suggested. ${ }^{1-4}$ Defects arising from resection of tumors involving the posterior part of the hard palate and posterolateral maxilla can be classified as limited type Ila defects, as per the classification suggested by Brown and Shaw. These defects are commonly reconstructed using an obturator, temporalis myofascial flap, or a radial forearm free flap (RFFF). ${ }^{5}$ However, when such tumors extend to involve the infratemporal fossa (ITF), there arises a need to obliterate the resultant cavity. In these situations, such options may not be ideal as they do not provide adequate soft tissue. Failure to

received

June 24, 2013

accepted after revision

July 7, 2013

published online

March 24, 2014 obliterate ITF cavity often results in postoperative collection and secondary infection. Such a cavity could heal with fibrosis resulting in trismus. ${ }^{6}$ Anterolateral thigh flap (ALT) or rectus abdominis myocutaneous flap may be used, however, these flaps have a bulky oral component and tend to have a delayed flap mucosalization, thereby adversely affecting the functional outcome.

Adipofascial ALT has been described for reconstruction of both the tongue and the floor of mouth defects. ${ }^{7,8}$ This flap is also used for correcting head and neck contour deformities. The adipofascial ALT flap has multiple advantages. Adequate adipofascial tissue can be harvested to match the defect requirement. ALT flap is well studied and has consistent
Copyright $\odot 2014$ by Thieme Medical Publishers, Inc., 333 Seventh Avenue, New York, NY 10001, USA. Tel: +1(212) 584-4662.
DOI http://dx.doi.org/ 10.1055/s-0034-1371973. ISSN 1943-3875. 

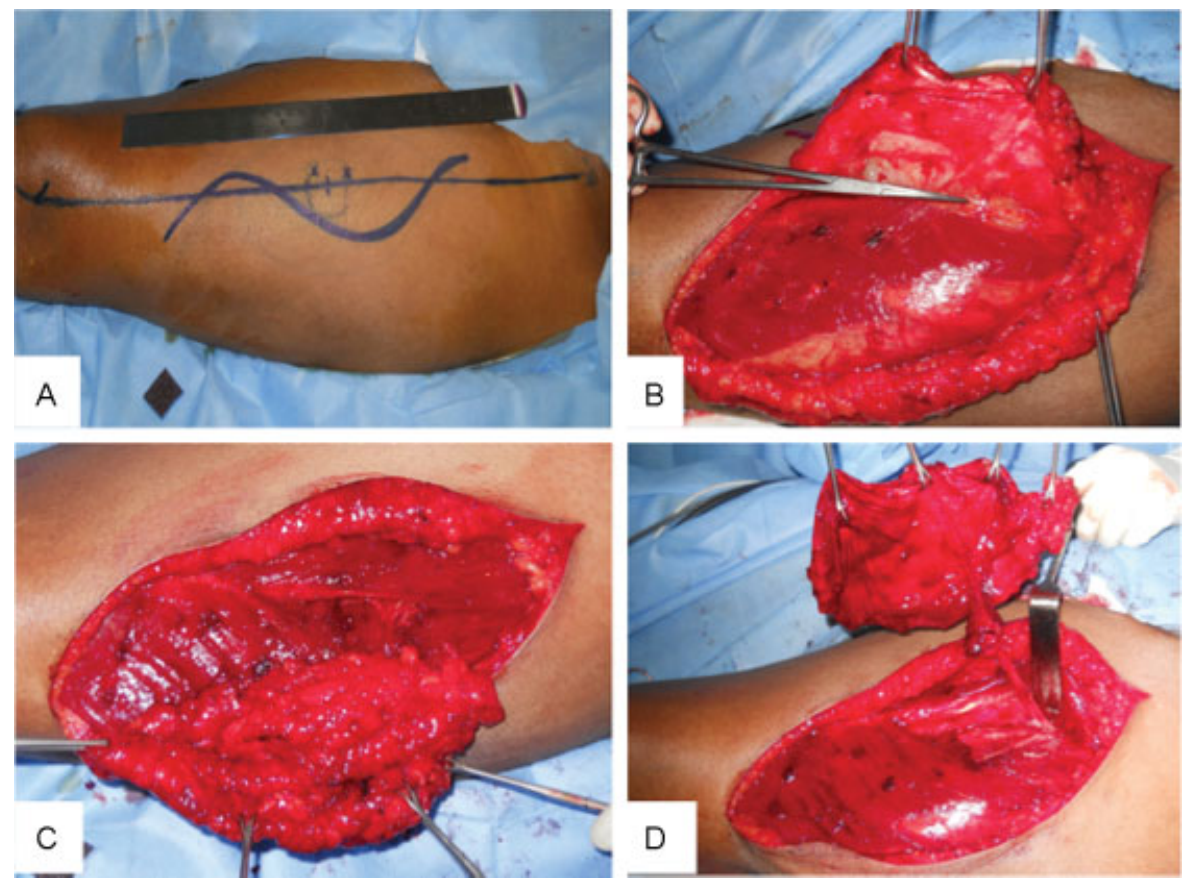

Figure 1 Harvesting an adipofascial anterolateral thigh flap. (A) Perforator and skin incision marking. (B) Identification of perforator from lateral approach. (C) Harvested adipofascial flap. (D) Perforator dissected through the muscle.

vascular anatomy. With adipofascial flap, there is no problem of hair growth and donor site can be closed primarily. However, application of this flap for reconstruction of maxillary defects has not been previously described. In this series, we describe the use of adipofascial ALT flap for reconstruction of type IIa maxillectomy defects with ITF extension and evaluate the associated complications as well as functional results.

\section{Patients and Methods}

Patients who underwent ALT flap reconstruction of maxillary defects at our center from August 2010 to July 2012 were identified using the database. Patients with maxillary defects reconstructed with adipofascial ALT were included in this study. All patients had infrastructural maxillectomy defects with extension into the ITF.
Flap technique: The techniques used for harvesting adipofascial ALT have been previously described. ${ }^{9}$ We modified the technique to preserve the skin at the donor site (-Fig. 1). An attempt was made to locate the perforator using a handheld Doppler. A Lazy-S incision is taken along the line joining anterior superior iliac spine and the lateral border of patella. The skin flaps are elevated just below the dermis in superficial subcutaneous plane. The fat and the fascia are then incised laterally and adipofascial flap is elevated over the vastus lateralis to identify the perforator. Following the identification of the perforator, a circumferential adipofascial incision was taken as per the flap requirement. The perforator was then traced in usual manner up to the pedicle. The skin was closed primarily over a suction drain.

Insetting: The flap edge closer to perforator was anchored first to stabilize the perforator and avoid any kinks and compressions. The flap edges were then sutured through

Table 1 Patient characteristics

\begin{tabular}{|l|l|l|l|l|}
\hline & Case 1 & Case 2 & Case 3 & Case 4 \\
\hline Age in y & 53 & 65 & 33 & 40 \\
\hline Gender & M & F & M & M \\
\hline Histology & ACC Palate & SCC palate & Ameloblastoma & ACC palate \\
\hline ITF involvement & Yes & Yes & Yes & Yes \\
\hline Mandibulotomy approach & Yes & No & Yes & Yes \\
\hline Post-op RT & Yes & No & No & Yes \\
\hline Local site complication & No & No & No & No \\
\hline
\end{tabular}

Abbreviations: ACC, adenoid cystic carcinoma; F, female; ITF, infratemporal fossa; M, male; RT, radiotherapy; SCC, squamous cell carcinoma. 
the fascia to oral cavity tissue. The remaining flap was folded and used to obliterate the ITF.

\section{Results}

Four patients who underwent maxillary defect reconstruction using adipofascial ALT were identified from the departmental database. The patient characteristics are detailed in - Table 1. Two patients had adenoid cystic carcinoma arising from the posterior hard palate with tumor tracking along the nerve to the ITF, requiring ITF clearance. One patient each had squamous cell carcinoma and ameloblastoma arising from hard palate and upper alveolus, respectively, with ITF involvement. None of the patients had tumor extending cranially toward the orbit floor. All patients had maxillary defects classified as type IIa associated with an ITF defect. Two patients had ipsilateral canine preserved while in one patient the defect extended up to the ipsilateral lateral incisor. Mandibulotomy approached was used in three patients for clearance of ITF disease. One patient had residual disease following primary radiotherapy for SCC of hard palate while the remaining three patients were previously untreated. Two patients received postoperative radiation.

The flap characteristics and outcome analysis are detailed in - Table 2. The mean flap size measured at the fascia was $150 \mathrm{~cm}^{2}$. All flaps were raised based on single perforator. In the three patients, the perforator was dissected through the vastus lateralis muscle while one patient had a septal perforator. In all three patients, the perforator was traced up to the descending branch, and in the fourth patient, up to the transverse branch of lateral circumflex femoral artery. All donor sites were closed primarily and none of the patients had donor-site complications. Facial artery was used as donor artery in three cases and superior thyroid artery in one. Venae comitantes were anastomosed end to side with internal jugular vein. None of the flaps were lost in this series.

All the adipofascial flaps mucosalized by 6 to 8 weeks. None of the patients had oroantral fistula. When ITF cavity is not obliterated, collection and secondary infections are common, and later, may result in trismus. In this series, the flap was used to obliterate the ITF cavity. None of the patients had any local complications. The mouth opening measured as the interincisal distance was more than $4 \mathrm{~cm}$. At a median followup of more than 1 year, all patients were taking a regular diet orally. Three patients had resumed regular oral diet, while one patient remained on a soft oral diet. A representative case is shown in -Fig. 2.

\section{Discussion}

The reconstruction of postablative midface defects is challenging. Various classifications have been proposed to develop a reconstruction algorithm. Type Ila defects usually involve oral lining defects with oroantral fistula. The goal of reconstructing such defects is to provide an oroantral separation and a smooth taut oral lining for dental prosthetic rehabilitation. When the defect does not extend to involve the incisors, a composite bone flap is usually not required. Multiple options are available for reconstruction of such defects; including a RFFF, submental flap, buccal pad of fat, temporalis myofascial flap, or an obturator flap. ${ }^{10}$ The RFFF is commonly used to provide an oral lining and to achieve oroantral separation. Studies have shown that RFFF has a functional advantage over obturator flap. ${ }^{11}$ In certain tumors involving the posterior part of hard palate, or maxillary tuberosity area, the tumor may extend to involve the masticator space. Complete removal of such tumors often results in a limited defect of palate and maxilla with a large cavity defect in the ITF. Obliteration of this cavity is essential to prevent immediate complications such as collection and secondary infections. In cases with extensive ITF dissection, the internal carotid artery is exposed and it becomes very important to provide a cover for the exposed vessel. Inadequate substitution of soft tissue may result in trismus. ${ }^{6}$ In such situations, reconstruction with a RFFF or obturator is inadequate. RFFF may not provide enough soft tissue to obliterate the ITF cavity. The temporalis flap cannot be used as its vascularity is compromised during ITF dissection.

A bulkier flap such as ALT or rectus abdominis myocutaneous free flaps may be considered. ALT is a versatile flap and has been used for oral cavity and maxillary reconstruction. ${ }^{12,13}$ ALT flap may provide adequate bulk, a part can be deepithelialized and used for obliterating the ITF defect. ${ }^{6}$ However, the intraoral skin paddle of ALT flap may be too

Table 2 Flap characteristics and outcome

\begin{tabular}{|l|l|l|l|l|}
\hline & Case $\mathbf{1}$ & Case 2 & Case 3 & Case 4 \\
\hline Size $\left(\mathrm{cm}^{2}\right)$ & 180 & 150 & 150 & 120 \\
\hline Number of perforators & 1 & 1 & 1 & 1 \\
\hline Perforator type & Muscular & Septal & Muscular & Muscular \\
\hline Pedicle & D & D & T & D \\
\hline Donor-site morbidity & None & None & None & None \\
\hline Mouth opening $>4 \mathrm{~cm}$ & Yes & Yes & Yes & Yes \\
\hline Oroantral fistula & No & No & No & No \\
\hline Oral diet & Yes & Yes & Yes & Yes \\
\hline
\end{tabular}

Abbreviations: D, descending branch; T, transverse branch. 

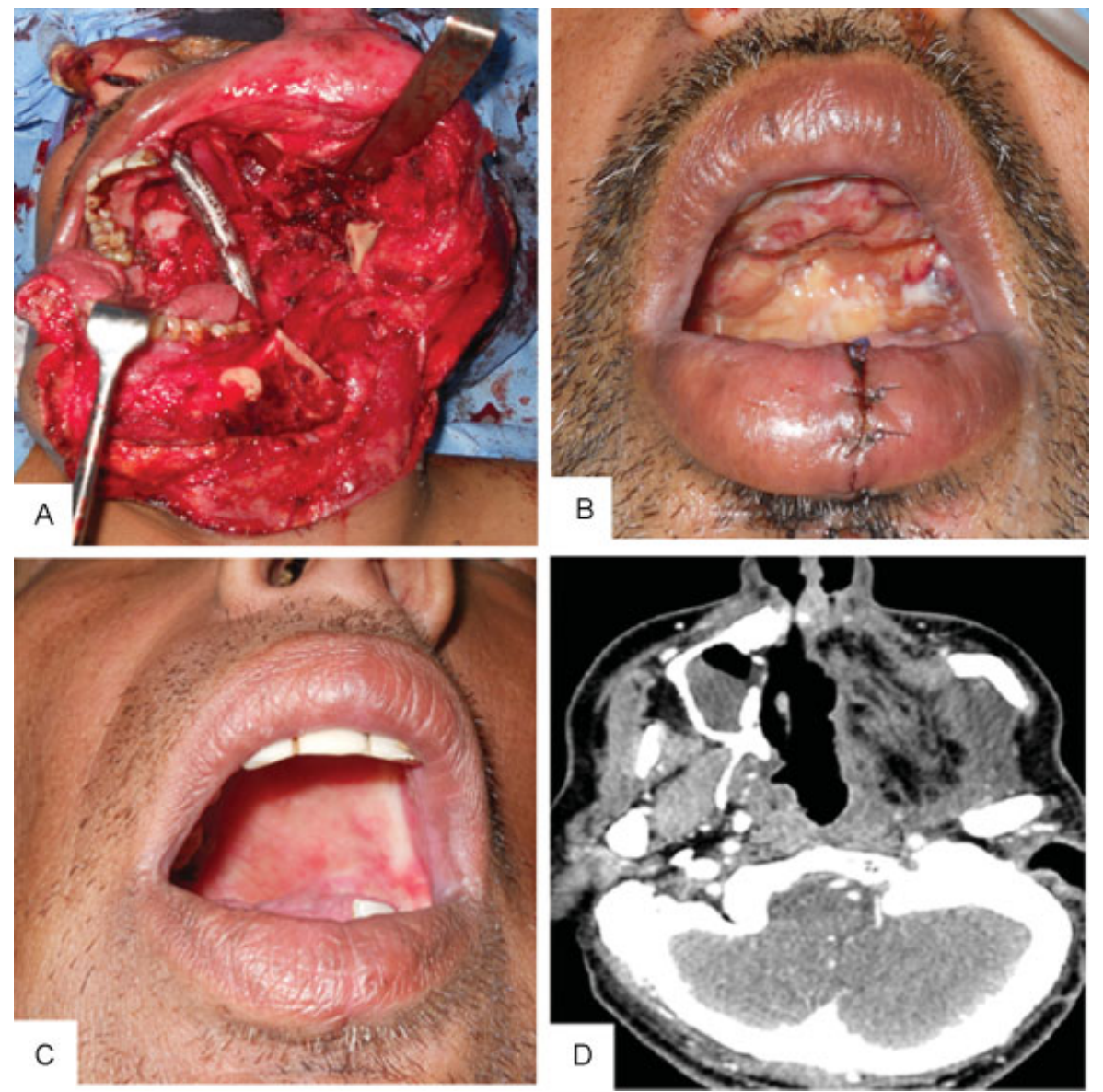

Figure 2 A representative case. (A) Defect involving posterior hard palate, posterolateral maxilla with extensive dissection of infratemporal fossa. (B) Flap in immediate postoperative period. (C) Good mouth opening and neo mucosalized flap at 3 months following surgery, (D) follow-up scan at 1 year show adipose tissue obliterating the infratemporal fossa.

bulky to provide a taut surface for dental rehabilitation. Also, there may be a problem of hair growth and the donor site may require skin graft. Rectus abdominis myocutaneous flap has also been extensively used for maxillary reconstruction particularly types III and IV defects. ${ }^{2,14}$ This flap can also be used for obliterating the ITF cavity. Similar to ALT, this flap may be bulky for oral cavity defect. Also, this flap may result in significant donor-site morbidity.

We describe the use of an adipofascial ALT flap for reconstruction of such defects. Adipofascial ALT has been previously described for other oral cavity subsites such as tongue and floor of mouth. ${ }^{9}$ This flap is also used for reconstruction of head and neck contour and extremity defects. ${ }^{15-17}$ In this study, we use this flap to provide oral lining and obliterate the ITF cavity. The intraoral part gradually shrinks and gets mucosalized providing a smooth taut surface for dental prosthesis. Consistent with the findings of previous study, our results also demonstrate adequate mucosalization of the flap. ${ }^{9}$ None of the patients had an oroantral fistula. All patients resumed oral feeds and had adequate mouth opening. The use of adipofascial RFFF has been described for type Ila defects ${ }^{18}$; however, this flap cannot be used in cases where the soft tissue requirement is significant. We recommend adipofascial ALTover adipofascial RFFF even in Type Ila defects due to several advantages. ALT has an advantage, that no major vessel is harvested along with the flap. The flap dimension and thickness can be adjusted according to the defect requirement and the adipose tissue can be thinned as required. Unlike radial forearm flap, the ALT scar can be easily concealed.

Previous studies reported harvesting ALT flap along with skin and subsequently discarding the skin to make it adipofascial. ${ }^{7}$ However, the flap can be harvested without taking the overlying skin as described in the method section. The donor site can be closed primarily thereby reducing the donor-site morbidity.

This flap may not be a good option when the defect extends to involve the adjoining buccal mucosa. As the flap shrinks considerably, the sulcus might get obliterated leading to problems with prosthetic rehabilitation, requiring secondary procedures. We also recommend early rehabilitation before obliteration of the vestibule. Also, when the defect involves the premaxilla a composite flap should be preferred. The limited number of patients in this series indicates that tumors with extensive ITF involvement but limited maxillary involvement are relatively rare.

In conclusion, adipofascial ALT is an excellent choice for limited defects of posterior hard palate and posterolateral 
maxilla, when there is a requirement for large soft tissue bulk to fill the ITF defect. The flap gets mucosalized and soft tissue obliterates ITF resulting in good functional outcome.

\section{Conflict of Interests}

None.

\section{References}

1 Brown JS, Shaw RJ. Reconstruction of the maxilla and midface: introducing a new classification. Lancet Oncol 2010;11(10): 1001-1008

2 Cordeiro PG, Santamaria E. A classification system and algorithm for reconstruction of maxillectomy and midfacial defects. Plast Reconstr Surg 2000;105(7):2331-2346, discussion 2347-2348

3 Okay DJ, Genden E, Buchbinder D, Urken M. Prosthodontic guidelines for surgical reconstruction of the maxilla: a classification system of defects. J Prosthet Dent 2001;86(4):352-363

4 Yamamoto Y, Kawashima K, Sugihara T, Nohira K, Furuta Y, Fukuda S. Surgical management of maxillectomy defects based on the concept of buttress reconstruction. Head Neck 2004;26(3):247-256

5 Futran ND, Mendez E. Developments in reconstruction of midface and maxilla. Lancet Oncol 2006;7(3):249-258

6 Kekatpure VD, Manjula BV, Mathias S, Trivedi NP, Selvam S, Kuriakose MA. Reconstruction of large composite buccal defects using single soft tissue flap-analysis of functional outcome. Microsurgery 2013;33(3):184-190

7 Agostini V, Dini M, Mori A, Franchi A, Agostini T. Adipofascial anterolateral thigh free flap for tongue repair. Br J Plast Surg 2003; 56(6):614-618
8 Agostini T, Agostini V. Adipofascial versus fasciocutaneous anterolateral thigh flap in oral cavity reconstruction. Focus on the vascular supply. J Plast Reconstr Aesthet Surg 2009;62(12): e633-e634

9 Agostini T, Agostini V. Further experience with adipofascial ALT flap for oral cavity reconstruction. J Plast Reconstr Aesthet Surg 2008;61(10):1164-1169

10 Dalgorf D, Higgins K. Reconstruction of the midface and maxilla. Curr Opin Otolaryngol Head Neck Surg 2008;16(4):303-311

11 Genden EM, Wallace DI, Okay D, Urken ML. Reconstruction of the hard palate using the radial forearm free flap: indications and outcomes. Head Neck 2004;26(9):808-814

12 Bianchi B, Ferri A, Ferrari S, Copelli C, Sesenna E. Maxillary reconstruction using anterolateral thigh flap and bone grafts. Microsurgery 2009;29(6):430-436

13 Kekatpure VD, Trivedi NP, Shetkar G, Manjula BV, Mathan Mohan A, Kuriakose MA. Single perforator based anterolateral thigh flap for reconstruction of large composite defects of oral cavity. Oral Oncol 2011;47(6):517-521

14 Bianchi B, Bertolini F, Ferrari S, Sesenna E. Maxillary reconstruction using rectus abdominis free flap and bone grafts. Br J Oral Maxillofac Surg 2006;44(6):526-530

15 Hanasono MM, Skoracki RJ, Silva AK, Yu P. Adipofascial perforator flaps for "aesthetic" head and neck reconstruction. Head Neck 2011;33(10):1513-1519

16 Wang X, Qiao Q Liu Z, et al. Free anterolateral thigh adipofascial flap for hemifacial atrophy. Ann Plast Surg 2005;55(6):617-622

17 Hsieh CH, Yang CC, Kuo YR, Tsai HH, Jeng SF. Free anterolateral thigh adipofascial perforator flap. Plast Reconstr Surg 2003; 112(4):976-982

18 Thankappan K, Trivedi NP, Sharma M, Kuriakose MA, Iyer S. Free radial forearm adiposo-fascial flap for inferior maxillectomy defect reconstruction. Indian J Plast Surg 2009;42(1):100-103 